\title{
Trypan blue- and indocyanine green- assisted epiretinal membrane surgery: clinical and histopathological studies
}

${ }^{1}$ Department of Ophthalmology Hong Kong Sanatorium and Hospital, Hong Kong People's Republic of China

${ }^{2}$ Department of Ophthalmology and Visual Sciences

Hong Kong

People's Republic of China

${ }^{3}$ Department of Anatomy The Chinese University of Hong Kong

Hong Kong Eye Hospital Hong Kong

People's Republic of China

Correspondence:

AKH Kwok

Department of

Ophthalmology

Hong Kong Sanatorium and Hospital

2 Village Road

Happy Valley

Hong Kong

Tel: +85228358890

Fax: + 85228358008

E-mail: alvinkwok@hksh.com

Received: 26 September 2003

Accepted: 28 November 2003

Published online: 5 March 2004

Financial interest/support: None
Abstract

Purpose To evaluate the clinical outcome and electron microscopic findings of trypan blue (Tb) and indocyanine green (ICG) assisted epiretinal membrane (ERM) surgery.

Methods This is a prospective consecutive noncomparative interventional case series. After pars plana vitrectomy, $0.1 \mathrm{ml}$ of $0.6 \mathrm{mg} / \mathrm{ml}$ Tb solution was applied for $1 \mathrm{~min}$ under air for ERM staining. After ERM removal, internal limiting membrane (ILM) was further peeled after staining with $0.2 \mathrm{ml}$ of $1 \mathrm{mg} / \mathrm{ml}$ ICG solution. Intraoperative specimens were sent for electron microscopy. Tb was considered useful if the edge of ERM was stained where peeling could be initiated with a clearer visualisation of the overall extent of the ERM.

Results In all, 16 eyes from 16 patients were recruited. There were nine grade 1 ERMs, five grade 2 ERMs, and two grade 3 ERMs. Tb was useful in six $(67 \%)$ of the nine eyes with grade 1 ERMs and in all eyes with grade 2 or 3 ERMs. The three remaining grade 1 ERMs were removed together with surrounding ILM that was stained by ICG. The mean line of improvement was 1.3 lines with the median BCVA improved from 6/12 to 6/9. All 16 eyes had symptomatic improvement and none developed ERM recurrence. No complication related to $\mathrm{Tb}$ or ICG was observed clinically or angiographically. Electron microscopy of the Tb-stained ERM specimens showed fragments of ILM in all specimens.

Conclusions $\mathrm{Tb}$ and ICG are useful intraoperatively to improve the visualisation and facilitate complete removal of ERM and ILM in macular ERM surgery.
AKH KWok ${ }^{1,2}$, TYY Lai ${ }^{2}$, WWY Li' ${ }^{2}$ DTW Yew ${ }^{3}$ and VWY Wong ${ }^{2}$

Eye (2004) 18, 882-888. doi:10.1038/sj.eye.6701359 Published online 5 March 2004

Keywords: epiretinal membrane, indocyanine green, macula, trypan blue

\section{Introduction}

Pars plana vitrectomy has been used since the 1970s for removal of epiretinal membrane (ERM) in patients with symptomatic visual disturbances. ${ }^{1}$ Since then, it has become a wellestablished vitreoretinal surgical procedure with most patients having favourable visual outcome postoperatively. ${ }^{2-7}$ However, in certain circumstances, recurrence of ERM may redevelop after initial surgical success. The rate of idiopathic ERM recurrence has been documented to be around $10 \%$, with reoperative rate at about $3 \% .{ }^{78}$ In certain subgroups like ERM in young patients, recurrence rates as high as $20 \%$ have been reported. ${ }^{9-11}$ Failure to completely remove the ERM may be one of the factors for ERM recurrence. ${ }^{3,12}$ As the ERM is usually a transparent or semitransparent friable membrane, techniques to enhance its visualisation may be beneficial in facilitating its complete removal and decreasing its recurrence after ERM surgery.

Trypan blue $(\mathrm{Tb})$ has been used as an intraoperative stain to facilitate anterior segment surgery. ${ }^{13-15}$ The safety of intravitreal application of $\mathrm{Tb}$ has also been demonstrated in rabbit studies. ${ }^{16}$ Recently, $\mathrm{Tb}$ has been shown to be a useful intraoperative agent for improving the visualisation and complete removal of peripheral ERM in patients with proliferative 
vitreoretinopathy. ${ }^{17} \mathrm{~Tb}$ has also been suggested to be useful in staining macular ERM and internal limiting membrane (ILM) in cases of macular hole and macular pucker. ${ }^{18}$

Another commonly used ophthalmic dye, indocyanine green (ICG), has been shown to stain and facilitate the removal of ILM around macular holes with good anatomical and visual outcomes. ${ }^{19-23}$ It has also been used in surgery for proliferative vitreoretinopathy and retinal detachment to facilitate ERM and ILM removal. ${ }^{24-26}$ In order to improve the visualisation of both the ERM and ILM, the use of a double-staining technique using $\mathrm{Tb}$ followed by ICG may facilitate ERM and ILM removal in macular pucker surgery. Owing to the potential retinal pigment epithelial toxicity associated with the use of hypo-osmotic ICG solution, Stalmans et $a l^{27}$ have described the use of double staining with $\mathrm{Tb}$ and iso-osmotic infracyanine green instead of ICG. We have previously demonstrated the usefulness of isoosmotic ICG dye in ILM peeling for various macular surgeries. ${ }^{21,22}$ The purpose of our current study is to determine the safety and efficacy of double staining using $\mathrm{Tb}$ and iso-osmotic ICG dyes in various grades of ERM in conventional macular ERM surgery that was not complicated by proliferative vitreoretinopathy.

\section{Patients and methods}

This study was a prospective noncomparative interventional consecutive case series conducted at Hong Kong Eye Hospital, Hong Kong. Consecutive patients of at least 18 years old scheduled for macular ERM surgery were prospectively recruited. Institutional ethical approval as well as informed consent from all patients were obtained. After routine examination, pupils were fully dilated and slit-lamp biomicroscopy was performed with a contact lens. Macular ERM was defined as a membrane causing wrinkling of the macula. The membrane was classified into three grades. ${ }^{21}$ Grade 1 ERM consisted of cellophane membrane causing irregular wrinkling of the inner retina with no edge of ERM seen elevated from the retina. Grade 2 ERM was more substantial ERM with full-thickness retinal distortion and the edge of ERM seen elevated from the retina, and the opaque part of membrane less than onehalf of the area of the ERM. Grade 3 ERM was thick opaque membrane with marked obscuration and distortion of underlying retina and vasculature, together with the opaque part of membrane at least one-half of the area of ERM. Cases of ERM that occurred in eyes with concurrent retinal detachment or with proliferative vitreoretinopathy were excluded. Indications for ERM surgery included decreased visual acuity, metamorphopsia, or monocular diplopia.
All operations were performed by a single surgeon (Dr Kwok). Tb solution was prepared according to Feron et al. ${ }^{17}$ After pars plana vitrectomy and removal of posterior hyaloid, a volume of $0.1 \mathrm{ml}$ of $0.6 \mathrm{mg} / \mathrm{ml} \mathrm{Tb}$ solution (VisionBlue; Dutch Ophthalmic Research Center, Zuidland, Netherlands) was gently injected over the disc and then the macula after one stage air/fluid exchange. After $1 \mathrm{~min}$, Tb solution was removed before resumption of infusion. The extent to which the ERM was stained or visually enhanced was noted. Tb was considered useful if a surgical edge of the ERM was stained in which peeling of ERM could be initiated and allowed a clearer visualisation of the overall extent of the ERM. Removal of the ERM was then performed. Afterwards, $0.2 \mathrm{ml}$ of $1 \mathrm{mg} / \mathrm{ml}$ ICG solution (299 mOsm) was applied for $30 \mathrm{~s}$ after the infusion temporarily stopped in order to stain any residual ILM. The ILM was then removed up to within a disc-diameter from the temporal vascular arcade and the nasal optic disc margin. The preparation of ICG and technique of ILM peeling has been described previously. ${ }^{19,20,22}$

Specimens of ERM and ILM removed were fixed in $2.5 \%$ glutaraldehyde in $0.1 \mathrm{M}$ phosphate buffer for $24 \mathrm{~h}$ at $4{ }^{\circ} \mathrm{C}$. They were then postfixed in $1 \%$ osmic tetroxide, dehydrated in a series of ethanol and cleared in propylene oxide, and finally embedded in Epon 812. Ultrathin $(70 \mathrm{~nm})$ sections were obtained

(Ultramicrotome S, Leica, Germany) and stained with uranyl acetate and lead citrate.

Preoperative data including age and sex of the patients, duration and nature of the ERM, types of symptoms, and the lens status were noted. Preoperative best-corrected Snellen's visual acuity (BCVA) was recorded by certified optometrists with a standard Snellen chart. Pre- and postoperative fluorescein angiography (FA) was performed to detect any potential toxicity associated with $\mathrm{Tb}$ or ICG. Intraoperative data including any concurrent surgical procedures and any intraoperative complications were noted. Postoperative data including extent of symptoms improvement, anatomical status of the macula, any postoperative complications, and BCVA at the last follow-up were recorded. Data analysis was performed using a statistical software (SPSS for Windows v.10.0, SPSS Inc., Chicago, IL, USA).

\section{Results}

\section{Patients' demographics}

A total of 16 eyes in 16 patients were recruited (Table 1). There were 12 female subjects and the mean age of the patients was 62.3 years (range, $46-72$ years). The numbers of grade 1, 2 and 3 ERMs were nine (56\%), five 
Table 1 Demographics of 16 patients underwent Tb- and ICG-assisted macular ERM surgery

\begin{tabular}{|c|c|c|c|c|c|c|c|c|c|c|c|c|c|c|c|}
\hline No. & Sex & Age & Eye & Diagnosis & Grade & $\begin{array}{c}\text { Follow-up } \\
\text { (months) }\end{array}$ & $\begin{array}{c}\text { Duration of } \\
\text { symptoms } \\
\text { (months) }\end{array}$ & $\begin{array}{l}\text { Preop. } \\
\text { lens } \\
\text { status }\end{array}$ & $\begin{array}{c}\text { Combine } \\
\text { phaco }+I O L\end{array}$ & $\begin{array}{l}\text { Preop. } \\
\text { BCVA }\end{array}$ & $\begin{array}{l}\text { Final } \\
B C V A\end{array}$ & $\begin{array}{c}\text { Change in } \\
\text { lines }\end{array}$ & $\begin{array}{c}\text { Symptoms } \\
\text { improved } \\
(\%)\end{array}$ & $\begin{array}{c}T b \\
\text { useful }\end{array}$ & $\begin{array}{l}\text { Residual } \\
\text { ILM } \\
\text { peeled } \\
\text { with } \\
\text { ICG }\end{array}$ \\
\hline 1 & $\mathrm{~F}$ & 72 & $\mathrm{~L}$ & Primary ERM + cataract & 2 & 9 & 24 & Phakic & Yes & $6 / 60$ & $6 / 60$ & 0 & 35 & Yes & Yes \\
\hline 2 & $\mathrm{~F}$ & 53 & $\mathrm{R}$ & Primary ERM + cataract & 3 & 9 & 9 & Phakic & Yes & $6 / 90$ & $6 / 48$ & 2 & 25 & Yes & Yes \\
\hline 3 & $\mathrm{~F}$ & 56 & $\mathrm{R}$ & Primary ERM & 1 & 7 & 4 & Phakic & No & $6 / 12$ & $6 / 12$ & 0 & 30 & No & Yes \\
\hline 4 & M & 67 & $\mathrm{~L}$ & Primary ERM & 1 & 7 & 8 & Phakic & Yes & $6 / 60$ & $6 / 18$ & 4 & 20 & Yes & Yes \\
\hline 5 & $\mathrm{~F}$ & 66 & $\mathrm{~L}$ & Primary ERM & 1 & 7 & 4 & Phakic & Yes & $6 / 9$ & $6 / 9$ & 0 & 100 & No & Yes \\
\hline 6 & $\mathrm{~F}$ & 52 & $\mathrm{~L}$ & Primary ERM & 2 & 7 & 7 & Phakic & No & $6 / 18$ & $6 / 6$ & 4 & 20 & Yes & Yes \\
\hline 7 & $\mathrm{~F}$ & 70 & $\mathrm{~L}$ & Primary ERM & 2 & 7 & 36 & PCIOL & No & $6 / 9$ & $6 / 9$ & 0 & 10 & Yes & Yes \\
\hline 8 & $\mathrm{~F}$ & 60 & $\mathrm{R}$ & Primary ERM + cataract & 1 & 6 & 18 & Phakic & Yes & $6 / 24$ & $6 / 7.5$ & 6 & 70 & Yes & No \\
\hline 9 & $\mathrm{~F}$ & 57 & $\mathrm{~L}$ & Primary ERM + cataract & 1 & 6 & 24 & Phakic & Yes & $6 / 9$ & $6 / 9$ & 0 & 50 & Yes & No \\
\hline 10 & $\mathrm{M}$ & 72 & $\mathrm{~L}$ & Primary ERM + cataract & 1 & 6 & 24 & Phakic & Yes & $6 / 18$ & $6 / 18$ & 0 & 40 & No & Yes \\
\hline 11 & $\mathrm{M}$ & 66 & $\mathrm{R}$ & Primary ERM & 2 & 5 & 60 & Phakic & Yes & $6 / 9$ & $6 / 7.5$ & 1 & 25 & Yes & Yes \\
\hline 12 & $\mathrm{~F}$ & 46 & $\mathrm{R}$ & Primary ERM & 1 & 5 & 5 & Phakic & Yes & $6 / 9$ & $6 / 7.5$ & 1 & 25 & Yes & Yes \\
\hline 13 & $\mathrm{~F}$ & 54 & $\mathrm{R}$ & Primary ERM & 1 & 5 & 36 & Phakic & Yes & $6 / 9$ & $6 / 9$ & 0 & 50 & Yes & Yes \\
\hline 14 & $\mathrm{~F}$ & 71 & $\mathrm{~L}$ & Primary ERM & 2 & 4 & 2 & PCIOL & No & $6 / 12$ & $6 / 12$ & 0 & 50 & Yes & No \\
\hline 15 & $\mathrm{~F}$ & 67 & $\mathrm{R}$ & Secondary ERM & 3 & 4 & 8 & Phakic & Yes & $6 / 48$ & $6 / 24$ & 2 & 35 & Yes & Yes \\
\hline 16 & M & 67 & $\mathrm{~L}$ & Primary ERM & 1 & 4 & 12 & PCIOL & No & $6 / 12$ & $6 / 9$ & 1 & 60 & Yes & Yes \\
\hline
\end{tabular}
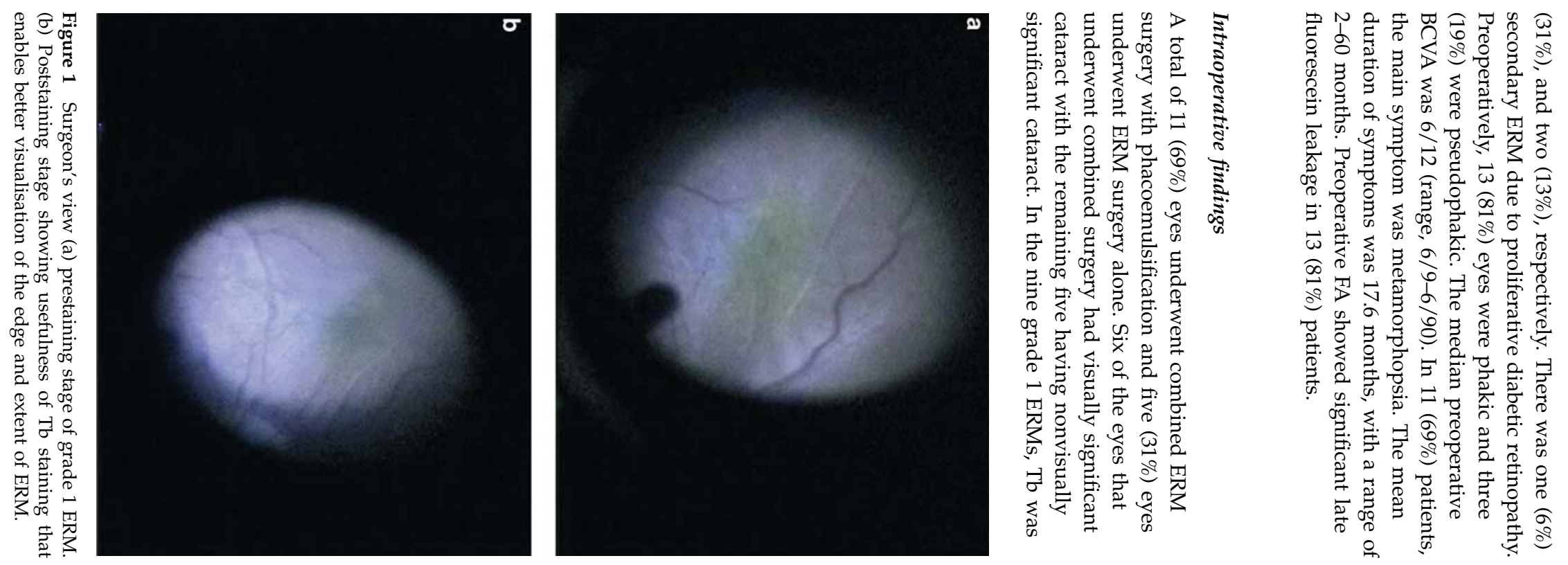
considered useful in staining of six $(67 \%)$ eyes (Figure 1) and was not useful in the other three $(33 \%)$ cases. These three ERMs were then removed together with surrounding ILM that was stained by ICG. Tb was useful in staining all grade 2 (Figure 2) and grade 3 ERMs. Grade 1 ERMs were less successfully stained with $\mathrm{Tb}$ compared with grade 2 and 3 ERMs, but the difference was not statistically significant $(P=0.21$, Fisher's exact test). Complete removal of ERMs was achieved in all cases. In three (19\%) patients, macular ILM was removed completely together with ERM after Tb staining (Figure 2d), as subsequent ICG staining did not reveal any residual macular ILM left. In the other 13 (81\%) eyes, remaining macular ILMs were found after ICG staining and removed subsequently.

\section{Postoperative findings}

The mean follow-up duration was 6.1. months (range, 4-9 months). At the last follow-up, the median postoperative BCVA was $6 / 9$ (range, 6/6-6/60), with the mean BCVA improvement of 1.3 lines (range, 0-6 lines). The final BCVA improved in five $(31 \%)$ eyes by two or more lines, while the others improved one line. Patients' subjective improvement in metamorphopsia ranged from 10 to $100 \%$, with a mean of $40 \%$. The mean lines of BCVA improvement was similar between patients that underwent combined surgery and ERM surgery alone $(P=0.66$, Mann-Whitney U test). In six $(46 \%)$ of the postoperative FA, a significant decrease in late fluorescein leakage was observed compared with the preoperative FA.

\section{Electron microscopic findings}

Surgical specimens from nine eyes were processed for electron microscopy. For the Tb-stained ERM specimens, all of them showed ILM fragments. One specimen (case no. 8) showed a small cellular fragment of Muller cell on the rough retinal side of ILM (Figure 3). For the three ICG-stained specimens of which both the ERM and ILM were intentionally removed together after failed $\mathrm{Tb}$ staining, one specimen (case no. 10) showed a small amount of cellular debris and fragments of plasma membrane of Muller cell on the rough retinal side of ILM (Figure 4). There were six ICG-stained ILM specimens retrieved after Tb-stained ERM removal. One of them showed a small amount of cellular debris on the rough retinal side of ILM (case no. 7, Figure 5); while another one had large fragments of Muller cell resembling cell processes and footplates on the rough retinal side of the ILM (case no. 2, Figure 6). No cellular fragment was present on the smooth vitreal side of ILM. No abnormal cellular debris on either the vitreal or the retinal side of ILMs was present in the remaining specimens.

From the limited amount of specimens obtained, specimens that were removed en bloc (ERM together with ILM) after ICG staining were compared with ILM
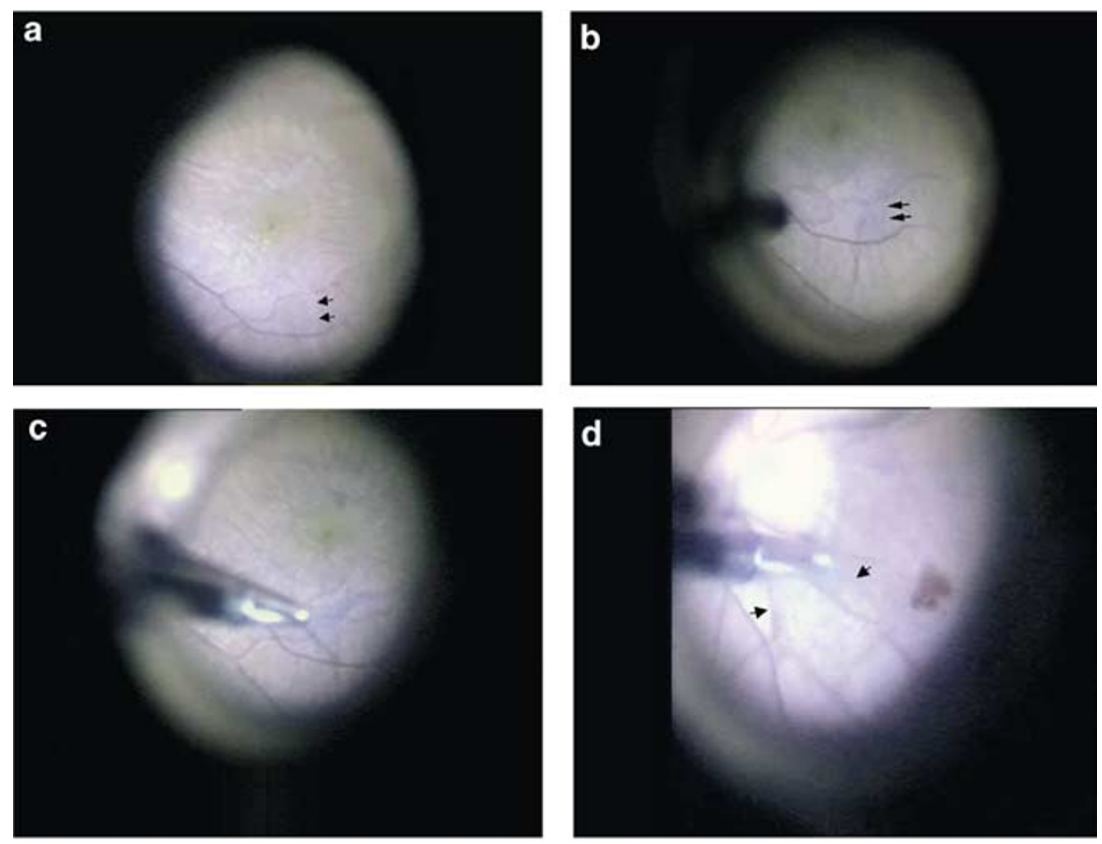

Figure 2 Surgeon's view (a) prestaining stage of grade 2 ERM, with the edge of ERM clearly stained where peeling could be started (arrows). (b) poststaining stage showing usefulness of Tb staining that enables better visualisation of the edge and extent of ERM. (c) surgical peeling of the stained ERM. (d) Tb-stained ILM being held and removed with an intraocular forceps (arrows). 


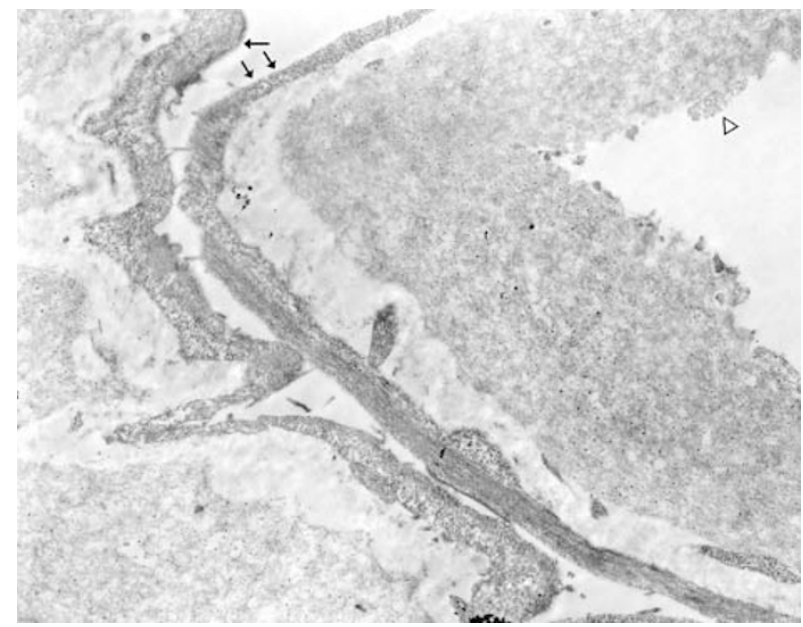

Figure 3 Electron microscopy of the Tb-stained ERM specimen (case no. 8). Layers of epiretinal cells (arrows) are seen on the smooth vitreal side of the ILM. A small cellular fragment of Muller cell (open arrow head) is observed on the rough retinal side of the ILM $(\times 8000)$.



Figure 4 Electron microscopy of the ICG-stained specimen of which the ERM and ILM are intentionally removed together (case no. 10). Previous staining with $\mathrm{Tb}$ has failed. Layers of epiretinal cells (arrows) are seen on the smooth vitreal side of the ILM. Only a small amount of cellular debris (open arrow head) is observed on the rough retinal side of the ILM. A cellular structure resembling fragments of plasma membrane of Muller cell is also seen (solid arrow head) $(\times 6000)$.

specimens that were obtained in two steps with sequential staining. Most of the specimens did not contain any cellular debris. In those rare cases where cellular debris was found, the en bloc specimen appeared to contain smaller cellular debris with only plasma membrane of Muller cells present on the retinal side of the ILM, while the ILM specimens obtained in two steps were found to have more cellular debris and larger fragments of Muller cells on the retinal side of the ILM.

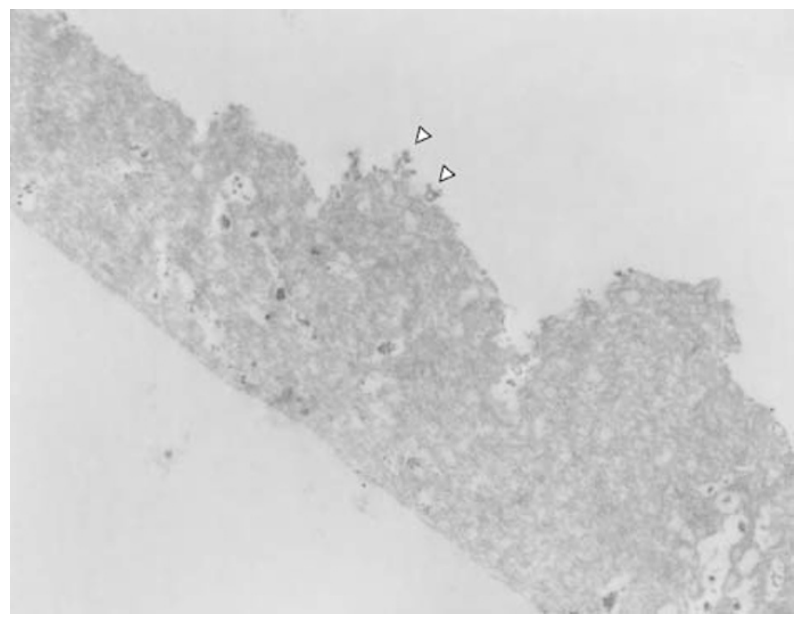

Figure 5 Electron microscopy of the ICG-stained ILM specimen (case no. 7). The ERM has been successfully removed with $\mathrm{Tb}$ staining before ILM removal. A small amount of suspicious cellular debris (open arrow heads) is observed on the rough retinal side of the ILM. The smooth vitreal side of the ILM is clean without cellular debris $(\times 8000)$.

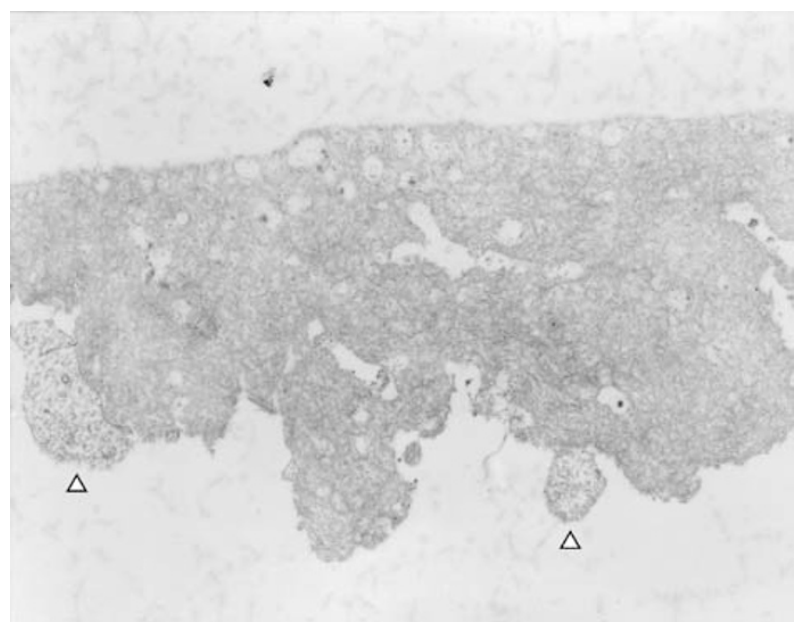

Figure 6 Electron microscopy of the ICG-stained ILM specimen (case no. 2). The ERM has been successfully removed with $\mathrm{Tb}$ staining before ILM removal. Large fragments of Muller cell (open arrow heads) resembling cell processes and footplates are observed on the rough retinal side of the ILM. The smooth vitreal side of the ILM is clean without cellular fragments $(\times 10000)$.

\section{Complications}

No intraoperative complication due to the use of $\mathrm{Tb}$ and ICG was observed in our study. One case developed postoperative angiographic cystoid macular oedema following combined phacoemulsification and ERM surgery. The patient had a final BCVA of $6 / 9$, which was the same as preoperative vision. No recurrence of ERM was observed. 


\section{Discussion}

Recurrence of ERM can lead to decreased visual acuity or recurrence of symptoms after initial successful ERM surgery. ${ }^{9}$ It may be attributed to incomplete removal of ERM during surgery. ${ }^{3,12}$ Since the ERM is usually a thin and semitransparent membrane, methods that enhance its visualisation and complete removal, especially in inconspicuous cellophane ERM, are desirable. Enhanced visualisation may also shorten the duration of surgery and minimise retinal trauma during ERM removal.

$\mathrm{Tb}$ is a vital stain that was said to be the preferred intraoperative dye during cataract surgery. ${ }^{14}$ No adverse effect related to the use of $\mathrm{Tb}$ has been observed in these cataract series. ${ }^{13-15}$ Recently, Feron et al ${ }^{17}$ demonstrated the usefulness of $\mathrm{Tb}$ in staining peripheral ERM in proliferative vitreoretinopathy. They did not find any ILM attached to ERM specimens. This is in contrast to our study in which all eyes had variable degree of ILM being removed together with ERM, as commonly reported in the literature. ${ }^{28,29}$ This may be due to different characteristics of macular and peripheral ERM, as well as idiopathic ERM and those associated with proliferative vitreoretinopathy.

In our study, we have demonstrated that $\mathrm{Tb}$ can effectively stain most ERMs and improve their visualisation intraoperatively. Tb staining of ERMs was particularly useful in grade 2 or 3 ERM in which the ERMs are thicker. For grade 1 ERM, Tb staining was less predictable with good staining achieved in six $(66 \%)$ of the nine cases. As grade 1 fine cellophane ERMs are more difficult to be visualised, methods that enhance their visualisation and removal would be desirable. These may be achieved by increasing the concentration or contact time of $\mathrm{Tb}$ instilled. Li et al ${ }^{18}$ successfully stained all ERMs with $0.5 \mathrm{ml} \mathrm{Tb}$ solution for $2 \mathrm{~min}$, as compared to $0.1 \mathrm{ml} \mathrm{Tb}$ solution for $1 \mathrm{~min}$ in ours, although they did not grade the ERMs. Similarly, the study by Stalman et $\mathrm{l}^{27}$ also successfully stained all ERMs with $0.2-0.5 \mathrm{ml} \mathrm{Tb}$ solution for $2 \mathrm{~min} .{ }^{27}$ Another option of removal of fine grade 1 ERMs is the use of ICG as demonstrated in our study. The ILM around ERM is stained by ICG and the two can be removed together during the removal of ILM. We have previously reported this technique in operating on a patient with grade 1 ERM that resulted in postoperative vision of $20 / 20$ and complete resolution of metamorphosia. ${ }^{30}$ Therefore, for grade 1 ERM, the use of $\mathrm{Tb}$ may not be required and ICG may be used instead.

Electron microscopy in our patients showed small amount of cellular debris with plasma membrane of Muller cell on the rough retinal side of ILM in one of the Tb-stained en bloc ERM and ILM specimens (case no. 8) (Figure 3). In two of the ICG-stained ILM specimens that were obtained in two steps with sequential staining, more cellular debris and larger fragments of Muller cells were observed (cases nos. 2 and 7) (Figures 5 and 6). The larger amount of cellular elements in the ILM specimens compared with the en bloc specimens may be due to closer contact of the dye in eyes with prior ERM removal as compared with those with ERM in situ, as the ERM that may act as a diffusion barrier for the dye to contact the retina. Despite the finding of the neuroglial tissue in the ERM and ILM specimens, it has been suggested that similar fragments might also be present in ERM specimens even without the use of dye staining. ${ }^{18}$ On the contrary to our electron microscopy findings, ruptured cells on both the vitreal and retinal sides of the ILM have been reported previously. ${ }^{31}$

Previous studies have demonstrated that removal of ILM may cause delayed recovery of focal macular electroretinogram b-wave after macular hole surgery that did not affect the visual acuity. ${ }^{32}$ Recent studies have also demonstrated that intraoperative use of hypo-osmotic ICG for ILM peeling in macular surgery may cause retinal damage by altering the cleavage plane to the innermost retinal layers and may result in less improvement of visual acuity and unexpected visual field defects. ${ }^{31,33}$ The exact mechanism of the possible ICG-related retinal damage is uncertain but may be related to the spectral absorption properties of ICG, causing a possible photodynamic effect of ICG at the vitreoretinal interface. ${ }^{34}$ Although we did not detect any toxicity related to $\mathrm{Tb}$ or ICG angiographically in this study, the safety of the dyes warrants further evaluation.

Fine ERM may occasionally cause significant metamorphopsia in patients with relatively good visual acuity. Early removal of the fine ERM may relieve the disturbing symptoms, and stabilise the vision that would possibly worsen later with less potential for recovery after vitrectomy. ${ }^{6}$ Detailed counselling about surgery of grade 1 ERM has to be carefully done. In our series, all 16 eyes including thin grade 1 ERM had symptomatic improvement postoperatively. This seems slightly superior to previous studies that around $80 \%$ of patients had improvement in vision postoperatively. ${ }^{2-6}$

This study had several limitations including a small sample size and the lack of control group for comparison. A longer follow-up is also required to demonstrate the long-term benefit of double-dye-assisted ERM and ILM peeling with Tb and ICG in preventing ERM recurrence.

In conclusion, we found that $\mathrm{Tb}$ and ICG were useful intraoperative dyes to facilitate visualisation and complete removal of ERM during macular surgery. Further studies on the safety, application timing, and the minimal effective concentration of $\mathrm{Tb}$ and ICG to be used in macular ERM surgery are warranted. 


\section{References}

1 Machemer R. A new concept for vitreous surgery. 7. Two instrument techniques in pars plana vitrectomy. Arch Ophthalmol 1974; 92: 407-412.

2 Michels RG. Vitrectomy for macular pucker. Ophthalmology 1984; 91: 1384-1388.

3 Margherio RR, Cox Jr MS, Trese MS, Murphy PL, Johnson J, Minor LA. Removal of epiretinal membranes. Ophthalmology 1985; 92: 1075-1083.

4 Rice TA, De Bustros S, Michels RG, Thompson JT, Debanne SM, Rowland DY. Prognostic factors in vitrectomy for epiretinal membranes of the macula. Ophthalmology 1986; 93: 602-610.

5 McDonald HR, Verre WP, Aaberg TM. Surgical management of idiopathic epiretinal membranes. Ophthalmology 1986; 93: 978-983.

6 Pesin SR, Olk RJ, Grand MG, Boniuk I, Arribas NP, Thomas MA et al. Vitrectomy for premacular fibroplasias. Prognostic factors, long-term follow up, and time course of visual improvement. Ophthalmology 1991; 98: 1109-1114.

7 Grewing R, Mester U. Results of surgery for epiretinal membranes and their recurrences. Br J Ophthalmol 1996; 90: 323-326.

8 Donati G, Kapetanios AD, Pournaras CJ. Complications of surgery for epiretinal membranes. Graefes Arch Clin Exp Ophthalmol 1998; 236: 739-746.

9 Smiddy WE, Michels RG, Gilbert HD, Green WR. Clinicopathologic study of idiopathic macular pucker in children and young adults. Retina 1992; 12: 232-236.

10 Banach MJ, Hassan TS, Cox MS, Margherio RR, Williams GA, Garretson BR et al. Clinical course and surgical treatment of macular epiretinal membranes in young subjects. Ophthalmology 2001; 108: 23-26.

11 Benhamou N, Massin P, Spolaore R, Paques M, Gaudric A. Surgical management of epiretinal membrane in young patients. Am J Ophthalmol 2002; 133: 358-364.

12 Wilkinson CP. Recurrent macular pucker. Am J Ophthalmol 1979; 88: 1029-1031.

13 Melles GR, de Waard PW, Pameyer JH, Beekhuis HW. Trypan blue capsule staining to visualize the capsulorhexis in cataract surgery. J Cataract Refract Surg 1999; 25: 7-9.

14 Yetik H, Devranoglu K, Ozkan S. Determining the lowest trypan blue concentration that satisfactorily stains the anterior capsule. J Cataract Refract Surg 2002; 28: 988-991.

15 Jacob S, Agarwal A, Agarwal A, Agarwal S, Chowdhary S, Chowdhary $\mathrm{R}$ et al. Trypan blue as an adjunct for safe phacoemulsification in eyes with white cataract. J Cataract Refract Surg 2002; 28: 1819-1825.

16 Veckeneer M, van Overdam K, Monzer J, Kobuch K, van Marle W, Spekreijse H et al. Ocular toxicity study of trypan blue injected into the vitreous cavity of rabbit eyes. Graefes Arch Clin Exp Ophthalmol 2001; 239: 698-704.

17 Feron EJ, Veckeneer M, Parys-Van Ginderdeuren R, Van Lommel A, Melles GR, Stalmans P. Trypan blue staining of epiretinal membranes in proliferative vitreoretinopathy. Arch Ophthalmol 2002; 120: 141-144.

18 Li K, Wong D, Hiscott P, Stanga P, Groenewald C, McGalliard J. Trypan blue staining of internal limiting membrane and epiretinal membrane during vitrectomy: visual results and histopathological findings. $\mathrm{Br} J$ Ophthalmol 2003; 87: 216-219.
19 Kwok AK, Li WW, Pang CP, Lai TY, Yam GH, Chan NR et al. Indocyanine green staining and removal of internal limiting membrane in macular hole surgery: histology and outcome. Am J Ophthalmol 2001; 132: 178-183.

20 Kwok AK, Lai TY, Chan WM, Woo DC. Indocyanine green staining and removal of retinal internal limiting membrane in stage 3 or 4 macular hole surgery. Br J Ophthalmol 2003; 87: 71-74.

21 Kwok AK, Lai TY, Yew DT, Li WW. Internal limiting membrane staining with various concentrations of indocyanine green dye under air in macular surgeries. Am J Ophthalmol 2003; 136: 223-230.

22 Kwok AK, Lai TY. Internal limiting membrane removal in macular hole surgery of severely myopic eyes: a casecontrol study. Br J Ophthalmol 2003; 87: 885-889.

23 Kwok AK, Lai TY, Yuen KS, Tam BS, Wong VW. Macular hole surgery with or without indocyanine green stained internal limiting membrane peeling. Clin Exp Ophthalmol 2003; 31: 470-475.

24 Kusaka S, Hayashi N, Ohji M, Hayashi A, Kamei M, Tano Y. Indocyanine green facilitates removal of epiretinal and internal limiting membranes in myopic eyes with retinal detachment. Am J Ophthalmol 2001; 131: 388-390.

25 Sakamoto H, Yamanaka I, Kubota T, Ishibashi T. Indocyanine green-assisted peeling of the epiretinal membrane in proliferative vitreoretinopathy. Graefes Arch Clin Exp Ophthalmol 2003; 241: 204-207.

26 Park DW, Dugel PU, Garda J, Sipperley JO, Thach A, Sneed $\mathrm{SR}$ et al. Macular pucker removal with and without internal limiting membrane peeling: pilot study. Ophthalmology 2003; 110: $62-64$.

27 Stalman P, Feron EJ, Parys-Van Ginderdeuren R, Van Lommel A, Melles GR, Veckeneer M. Double vital staining using trypan blue and infracyanine green in macular pucker surgery. Br J Ophthalmol 2003; 87: 713-716.

28 Smiddy WE, Maguire AM, Green WR, Michels RG, de la Cruz Z, Enger $C$ et al. Idiopathic epiretinal membranes. Ultrastructural characteristics and clinicopathological correlation. Ophthalmology 1989; 96: 811-821.

29 Mittleman D, Green WR, Michels RG, de la Cruz Z. Clinicopathologic correlation of an eye after surgical removal of an epiretinal membrane. Retina 1989; 9: 143-147.

30 Kwok AKH, Lai TYY, Chan NR. Epiretinal membrane formation with internal limiting membrane wrinkling following Nd:YAG laser membranotomy in Valsalva retinopathy. Am J Ophthalmol 2003; 136: 763-766.

31 Haritoglou C, Gandorfer A, Gass CA, Schaumberger M, Ulbig MW, Kampik A. The effect of indocyanine-green on functional outcome of macular pucker surgery. Am J Ophthalmol 2003; 135: 328-337.

32 Terasaki H, Miyake Y, Nomura R, Piao CH, Hori K, Niwa T et al. Focal macular ERGs in eyes after removal of macular ILM during macular hole surgery. Invest Ophthalmol Vis Sci 2001; 42: 229-234.

33 Haritoglou C, Gandorfer A, Gass CA, Schaumberger M, Ulbig MW, Kampik A. Indocyanine green-assisted peeling of the internal limiting membrane in macular hole surgery affects visual outcome: a clinicopathologic correlation. Am J Ophthalmol 2002; 134: 836-841.

34 Gandorfer A, Haritoglou C, Gandorfer A, Kampik A. Retinal damage from indocyanine green in experimental macular surgery. Invest Ophthalmol Vis Sci 2003; 44: 316-323. 